\title{
Modificaciones neurofisiológicas de ondas beta durante un test atencional tras una intervención de ejercicio físico
}

\author{
Neurophysiological modifications of beta waves during an atencional'test after \\ an intervention of physical exercise

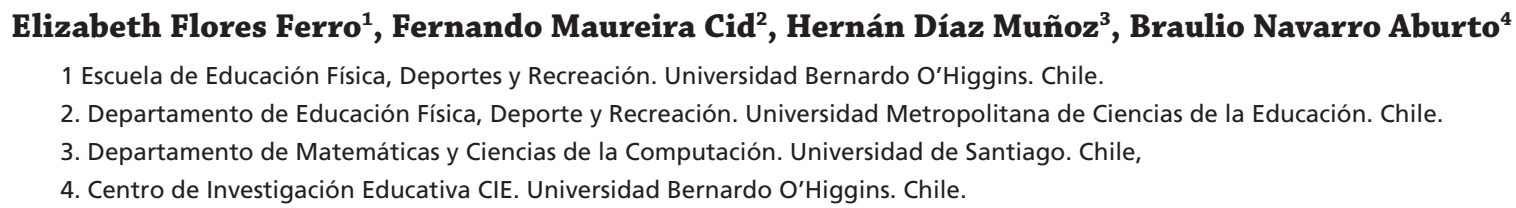

CORRESPONDENCIA:

Fernando Maureira Cid

maureirafernando@yahoo.es

Recepción: enero 2019 • Aceptación: diciembre 2019
CÓMO CITAR EL ARTÍCULO:

Flores, E., Maureira, F., Díaz-Muñoz, H., \& Navarro-Aburto, B. (2020). Modificaciones neurofisiológicas de ondas beta durante un test atencional tras una intervención de ejercicio físico. Cultura, Ciencia y Deporte, 15(44), 201-211.

\section{Resumen}

El objetivo del presente estudio fue conocer los efectos de una sesión de ejercicio físico aeróbico sobre la actividad eléctrica cerebral durante la resolución de una prueba de atención sostenida. Para ello se evaluó a 14 estudiantes universitarios varones, quienes resolvieron una prueba de atención antes y después de una intervención de 30 minutos de ejercicio físico aeróbico. Para registrar su actividad cerebral se utilizó el dispositivo cerebro-interfaz Emotiv Epoc®. Se analizaron los registros del lóbulo frontal, temporal y occipital en el rango de frecuencia de 13 a $30 \mathrm{~Hz}$ (onda beta). Los resultados muestran valores de índices de Hurst menores a 0,5, lo que muestra una actividad antipersistente, es decir, la actividad del cerebro cambia segundo a segundo durante la resolución de la prueba, situación que ocurre antes y después del ejercicio físico. Los sujetos tienden a presentar bajo número de correlaciones entre las regiones cerebrales estudiadas y parece ser que el ejercicio permite mantener un trabajo independiente de diversas regiones de la corteza, mejorando el desempeño en problemas de atención sostenida.

Palabras claves: ejercicio aeróbico, atención, electroencefalografía, índice de Hurst.

\begin{abstract}
The aim of the present study was to know the effects of a session of physical aerobic exercise on the electrical cerebral activity during the resolution of a test of supported attention. For it 14 university students were evaluated males, who solved a test of attention before and after an intervention of 30 minutes of physical aerobic exercise. To register his cerebral activity brain-interface was in use the device Emotiv Epoc®. There analyzed the records of the frontal, temporal and occipital lobe in the range of frequency from 13 to $30 \mathrm{~Hz}$ (beta wave). The results show values of indexes of minor Hurst to 0,5 what shows an antipersistent activity, that is to say, the activity of the brain changes second to second during the resolution of the test, situation that happens before and after the physical exercise. The subjects tend to have a low number of correlations between the brain regions studied and it seems that exercise allows to maintain an independent work of different regions of the cortex, improving performance in sustained attention's problems.
\end{abstract}

Key words: aerobic exercise, attention, electroencephalography, Hurst index. 


\section{Introducción}

La atención es un complejo sistema cerebral que nos permite seleccionar y focalizar nuestra percepción, captando activamente ciertos elementos del entorno desechando otros (Estévez, García, \& Junque, 1997; Fuenmayor \& Villasmil, 2008). Por su parte, para Maureira (2018) la atención se caracteriza por focalizar selectivamente las percepciones de cada persona, ello debido a la enorme cantidad de estímulos que el sistema nervioso constantemente está recibiendo, por lo que no puede procesar todo al mismo tiempo y finalmente debe elegir el o los estímulos más adecuados para cada situación. Para García (1997) los factores que determinan el proceso atencional son las particularidades del objeto, como por ejemplo el color, también influyen los niveles de activación fisiológica (predisposición del sistema nervioso ante una situación) y, finalmente, los aspectos psicológicos que corresponden a la motivación (intereses y expectativas del sujeto).

Actualmente se acepta la existencia de variados procesos atencionales, como el estado de alerta, la atención alternante, la atención dividida, la atención selectiva, la atención sostenida. (Maureira \& Flores, 2016). Esta última, se caracteriza por el mantenimiento de un foco atencional de manera voluntaria (también conocida como concentración). En relación con ello, los estudios de neuroimagen y las bases cerebrales de la atención sostenida se han centrado primordialmente en la corteza prefrontal y parietal superior, y en forma secundaria en el tálamo y la formación reticular colaboran en las tareas de control de activación cortical (Fan, McCandliss, Fossella, Flombaum \& Posner, 2005; Hirata \& Castro-Alamancos, 2010).

En el ámbito de la actividad física existen numerosos trabajos que muestran efectos benéficos del ejercicio físico sobre diversos tipos de atención: por ejemplo, Altenburg, Chinapaw \& Singh (2016) revelaron que 2 sesiones de 20 minutos antes y después de una clase de 90 minutos en niños de 10 a 13 años mejora significativamente los niveles de atención. Por otro lado, Iuliano, di Cagno, Aquino, Fiorilli, Mignogna, Calcagno, et al. (2015) realizaron una investigación interviniendo 12 semanas con ejercicio físico a una muestra de adultos mayores, mostraron que el entrenamiento cardiovascular disminuye significativamente en las respuestas perdidas de la prueba de atención dividida. Por su parte, Vidoni, Johnson, Morris, Van Sciver, Greer, Billinger, et al. (2015) evidenciaron que 26 semanas de actividad física en sujetos sedentarios produce mejoras en la atención simple y en el procesamiento visoespacial. Otro estudio realizado por Bullock y Giesbrecht (2014) revela que el ejercicio aeróbico mejora la atención en tareas visuales; a este trabajo se suman los de Hseih, Chang, Fang \& Hung (2016) y Rogerson \& Barton (2015), obteniendo resultados similares en la mejora de la atención tras la intervención del ejercicio físico. Finalmente, Alves, Tessaro, Teixeira, Murakava, Roschel, Gualano, et al. (2014) aplicaron un entrenamiento de intervalos de alta intensidad a 22 personas de mediana edad, los resultados mostraron mejora en la atención, sin embargo, no encontraron diferencias significativas en la memoria de corto plazo.

Estas mejoras se atribuyen a un aumento de la vascularización cerebral, neurogénesis, sinaptogénesis, aumento de la circulación sanguínea cerebral, etc. (Cotman \& Berchtold; 2002; Voss, Prakash, Erickson, Basak, Chaddock, Kim, et al., 2010).

Sin embargo, no existen trabajos que estudien los efectos del ejercicio físico sobre funciones cognitivas centrándose en la actividad eléctrica cerebral (Maureira \& Flores, 2018), sino, más bien, los estudios con electroencefalografía (EEG) se centran en describir diferencias entre deportistas de diversas disciplinas (Darocha, De Souza, Spindolab, Vaza, De Oliveira \& Geremiad, 2016), reducción de la actividad eléctrica cerebral tras la aplicación de una carga de trabajo físico (Ludyga, Gronwald \& Hottenrott, 2016) o diferencias en la actividad neuroeléctrica durante la ejecución de movimientos deportivos exitosos y errados (Babiloni, Infarinato, Marzano, Lacobani, Dassú, Soricelli, et al., 2011).

Los estudio con EEG se basan en que la actividad eléctrica cerebral es rítmica y se caracteriza por su frecuencia (cantidad de oscilaciones por segundo), que se mide en Hertz $(\mathrm{Hz})$, de tal forma que es posible diferenciar cinco tipos de ondas: a) Delta, que posee una frecuencia de $0.5-3 \mathrm{~Hz}$ y se observa durante las etapas de sueño profundo; b) Theta, con frecuencia de 3-7.5 $\mathrm{Hz}$ y se presenta en las etapas de sueño ligero; c) Alfa, que posee una frecuencia de $8-12 \mathrm{~Hz}$ y que caracteriza a un sujeto despierto, relajado con los ojos cerrados; d) Beta, que posee una frecuencia de $13-30 \mathrm{~Hz}$ y que se registra en un sujeto despierto percibiendo estímulos de entorno y resolviendo problemas cognitivos; e) gamma, que posee una frecuencia $>30 \mathrm{~Hz}$ y se relaciona con fenómenos de sincronización y estados de meditación (Maureira, 2017). Por lo anterior, la presente investigación utilizó la onda beta registrada por el EEG, ya que se relaciona con la resolución de problemas cognitivos como la atención.

La señal del EEG puede ser una herramienta importante para estudiar la actividad cerebral antes y después de una intervención con ejercicio físico, de manera de entregar más información que pueda explicar las causas de la mejora en pruebas cognitivas (atención, planificación, memoria, etc.) que se observa con la 
práctica de actividad física. Por eso el objetivo de la presente investigación es conocer los efectos de una sesión de ejercicio físico aeróbico sobre la actividad eléctrica cerebral durante la resolución de una prueba de atención sostenida.

\section{Material y métodos}

\section{Muestra}

Es de tipo no probabilística voluntaria, constituida por 14 varones estudiantes de la carrera de pedagogía en educación física de una universidad privada de Santiago de Chile. Los criterios de inclusión fueron que ninguno de los participantes presentara alteraciones cognitivas ni consumir algún fármaco que pudiese afectar el rendimiento en una prueba neuropsicológica. Además, que ninguno de los participantes presentara lesiones o trastornos que pudiesen afectar la realización de ejercicio físico. Ningún participante debía realizar un entrenamiento igual o superior a 3 horas semanales de alguna especialidad deportiva fuera del horario de clases de la carrera; de esta forma la muestra fue homogénea. La edad mínima fue de 18 años y la máxima de 21 , con una media de 19.7 \pm 1.2 . Todos los participantes firmaron un consentimiento informado.

\section{Instrumentos}

Para el registro EEG se utilizó el dispositivo cerebrointerfaz Emotiv Epoc ${ }^{\circledR}$ con frecuencia de muestreo de $128 \mathrm{~Hz}$. El EEG registra 14 canales a través de electrodos posicionados según el sistema 10/20 usando como referencia los electrodos del hueso mastoides. Los datos del EEG fueron procesados con el programa EEGLAB y ADJUST ejecutados en la plataforma MATLAB 2008. Se utilizaron los registros del lóbulo frontal (AF3 y AF4), temporal (T7 y T8) y occipital (O1 y O2). Se analizó el rango de frecuencia de 13 a $30 \mathrm{~Hz}$ (onda beta).

Para la evaluación de la atención sostenida se utilizó la prueba de Toulouse-Piéron, considerada como una de las técnicas más relevantes para evaluar la atención (León-Carrión, 1995). La prueba consta de una matriz de 1200 signos, distribuidos en 40 filas x 30 columnas. La prueba presenta dos figuras modelos en la parte superior de la hoja y la finalidad es encontrar las figuras que presentan las mismas características. El puntaje final corresponde al Índice Global de Atención y Percepción (IGAP) que se obtiene cuando al total de aciertos (A) se le resta la suma de errores (E) y omisiones (O). La aplicación de la prueba se realizó en forma individual, con una duración de ocho minutos.

\section{Procedimiento}

Del total, siete estudiantes fueron asignados aleatoriamente al grupo experimental y siete al grupo control. En la primera sesión se registró la frecuencia cardíaca basal de cada participante durante cinco minutos, durante los cuales permanecía recostado en una colchoneta. Posteriormente, cada sujeto realizó la prueba de atención durante ocho minutos, mientras se registraba su actividad cerebral con el EEG. En la segunda sesión, realizada siete días después, se volvió a registrar la frecuencia cardíaca basal y el grupo experimental realizó un trabajo aeróbico durante 30 minutos en una cinta rodante (BHF1 serie F) a una intensidad del 60\%-75\% de la frecuencia cardíaca máxima, obtenida individuamente con la fórmula de Karvonen: (FCmáx - FCr) * \% de trabajo + FCr; donde FCmáx es la frecuencia cardíaca máxima y $\mathrm{FCr}$ es la frecuencia cardíaca de reposo (Willmore \& Costill, 2004). Esto se controló con un dispositivo pectoral de medición de FC modelo Polar T31CODED. Una vez finalizada la intervención, se esperó a que el participante volviera a su frecuencia cardíaca basal y se le aplicó nuevamente la prueba de atención, registrando su actividad cerebral.

En la segunda sesión el grupo control realizó nuevamente la prueba de atención, registrando su actividad cerebral con el EEG.

Todas estas evaluaciones fueron realizadas en el Laboratorio de Neurofisiología, ejercicio físico y teoría del caos de la Universidad Católica Silva Henríquez de Chile.

\section{Plan de análisis de datos}

Para el registro y análisis de datos del electroencefalograma se utilizó el programa Matlab con la herramienta EEGlab. Para estudiar las series temporales del registro del EEG se utilizó el índice de Hurst, ya que es el mejor indicador del comportamiento caótico de un sistema a lo largo del tiempo (Pikovsky, Rosenblum \& Kurths, 2001) y desde hace algunos años se ha asumido la dinámica no lineal de las señales eléctricas del cerebro (Buzsaki, 2006). Este índice varía entre 0 y 1 , un valor $\mathrm{H}=0,5$ indica un comportamiento totalmente aleatorio del sistema, lo que hace imposible la predicción del comportamiento futuro. Un valor $0,5<\mathrm{H} \leq 1$ es indicador de un comportamiento persistente del sistema, es decir, la actividad pasada del sistema se sigue de otra igual. Un valor $0 \leq \mathrm{H}<0,5$ es indicador de un comportamiento antipersistente, es decir, la actividad pasada del sistema se sigue de una actividad opuesta (Díaz, Maureira \& Córdova, 2018). En la figura 1 se observa la fórmula del índice de Hurst. 


\section{$E\left[\frac{R(n)}{S(n)}\right]=C n^{H}$ as $n \rightarrow \infty$}

Figura 1. Índice de Hurst, donde $R(n)$ es el rango de las primeras $n$ desviaciones acumulativas de la media, $S(n)$ es su desviación estándar, $\mathrm{n}$ es el tiempo de observación y C es una constante (Díaz, et al., 2018).

Para la presente investigación se calculó el índice $\mathrm{H}$ para cada segundo de registro, obteniendo 480 índices de $\mathrm{H}$ (la prueba dura 480 segundos) y luego se obtiene el promedio de estos 480 valores. Esto se realizó para cada uno de los seis electrodos de cada sujeto evaluado. Una vez determinadas las medias de los índices $\mathrm{H}$ se utilizó el programa estadístico SPSS 24.0 para Windows. La prueba de normalidad de Kolmogorov-Smirnov (KS) entregó valores $p<0.05$ para cada registro de 480 índices $\mathrm{H}$, razón por la cual se utilizó estadística paramétrica para los análisis. Para comparar la actividad cerebral entre las cortezas prefrontales, temporales y occipitales antes y después de la intervención con ejercicio físico se utilizaron pruebas $t$ para muestras relacionadas. Para estudiar las relaciones entre diversas regiones cerebrales se utilizaron pruebas de correlación de Pearson. Finalmente, para los análisis comparativos de porcentajes en la variación de la actividad eléctrica cerebral se utilizaron pruebas de proporciones. Se consideraron significativos valores $\mathrm{p}<0.05$.

\section{Resultados}

En relación con los puntajes de la prueba de atención sostenida (IGAP) de Toulouse-Piéron, el grupo control no presenta diferencias significativas entre la primera y segunda medición $\left(\mathrm{X}_{1}=179.7 \pm 53.47\right.$; $\left.X_{2}=220.9 \pm 29.73 ; p=0.065\right)$. En cambio, en el grupo experimental se puede apreciar que existen diferencias significativas entre las mediciones pre y post ejercicio físico $\left(\mathrm{X}_{1}=172.6 \pm 41.59 ; \mathrm{X}_{2}=237.0 \pm 48.38 ; \mathrm{p}=0.011\right)$, lo que indica una mejora en la resolución de la prueba cognitiva tras la actividad física.

En las figuras 2 y 3 se observan las comparaciones de la actividad eléctrica utilizando el índice de Hurst de la corteza prefrontal durante las dos mediciones realizadas. Se puede apreciar que existen diferencias significativas en la región prefrontal izquierda (AF3) en el sujeto 2 ( $\mathrm{t}=4.896 ; p=0.000)$, el sujeto $4(\mathrm{t}=-6.890$; $p=0.000)$ y sujeto $7(\mathrm{t}=-5.362 ; p=0.000)$ del grupo control; y en el sujeto $8(\mathrm{t}=-3.657 ; p=0.000)$, sujeto 10 $(\mathrm{t}=9.711 ; p=0.000)$, sujeto $12(\mathrm{t}=6.960 ; p=0.000)$ y sujeto $14(\mathrm{t}=-6.274 ; p=0.000)$ de grupo experimental. El grupo control presentó modificaciones de los índices $\mathrm{H}$ prefrontales izquierdos en tres sujetos (42.9\%) en la segunda medición (dos aumentaron y uno disminuyo) y en el grupo experimental cuatros sujetos (57.1\%) mostraron modificaciones tras el ejercicio físico (dos aumentaron y dos disminuyeron), sin que existan diferencias significativas entre ambos grupos $(Z=-0.537)$.

En la región prefrontal derecha (AF4) existen diferencias significativas en el sujeto $1(t=2.721 ; p=0.007)$, sujeto $2(\mathrm{t}=4.896 ; p=0.000)$, sujeto $4 \quad(\mathrm{t}=-7.245$; $p=0.000)$, sujeto $6(\mathrm{t}=-4.977 ; p=0.000)$ y sujeto $7(\mathrm{t}=-$ 3.265; $p=0.001$ ) del grupo control, y el sujeto 8 ( $t=-$ $4.137 ; p=0.000)$, sujeto $9(\mathrm{t}=-9.083 ; p=0.000)$, sujeto $10(\mathrm{t}=5.111 ; p=0.000)$, sujeto $12(\mathrm{t}=6.141 ; p=0.000)$ y sujeto $14(\mathrm{t}=-5.484 ; p=0.000)$ del grupo experimental. El grupo control presentó modificaciones de los índices $\mathrm{H}$ prefrontales derechos en cinco sujetos (71.4\%) en la segunda medición (tres aumentaron y dos disminuyeron) y en el grupo experimental cinco sujetos (71.4\%) mostraron modificaciones tras el ejercicio físico (tres aumentaron y dos disminuyeron), sin que existan diferencias significativas entre ambos grupos $(\mathrm{Z}=0.0)$.

En las figuras 4 y 5 se observan las comparaciones de la actividad eléctrica utilizando el índice de Hurst de la corteza temporal durante las dos mediciones realizadas. Se puede apreciar que existen diferencias significativas en la región temporal izquierda (T7) en el sujeto 2 ( $\mathrm{t}=10.030 ; p=0.000)$, el sujeto 4 ( $\mathrm{t}=-5.278$; $p=0.000)$, el sujeto $6(\mathrm{t}=2.991 ; p=0.003)$ y el sujeto 7 $(\mathrm{t}=-4.806 ; p=0.000)$ del grupo control; $\mathrm{y}$ en el sujeto $9(\mathrm{t}=22.259 ; p=0.000)$, sujeto $10(\mathrm{t}=17.160 ; p=0.000)$, sujeto 12 ( $\mathrm{t}=16.454 ; p=0.000)$, sujeto 13 ( $\mathrm{t}=3.757$; $p=0.000)$ y sujeto $14(\mathrm{t}=-4.669 ; p=0.000)$ de grupo experimental. El grupo control presentó modificaciones de los índices $\mathrm{H}$ temporales izquierdos en cuatro sujetos $(57.1 \%)$ en la segunda medición (dos aumentaron y dos disminuyeron) y en el grupo experimental cinco sujetos $(71.4 \%)$ mostraron modificaciones tras el ejercicio físico (uno aumentó y cuatro disminuyeron), sin que existan diferencias significativas entre ambos grupos $(Z=-0.565)$.

En la región temporal derecha (T8) existen diferencias significativas en el sujeto 1 ( $\mathrm{t}=2.398 ; p=0.017)$, sujeto $2(\mathrm{t}=10.096 ; p=0.000)$ y sujeto $7(\mathrm{t}=-2.178$; $p=0.030)$ del grupo control, y el sujeto $8(\mathrm{t}=-9.942$; $p=0.000)$, sujeto $9(\mathrm{t}=4.380 ; p=0.000)$, sujeto 10 $(\mathrm{t}=6.788 ; p=0.000)$, sujeto $12(\mathrm{t}=11.438 ; p=0.000)$ $\mathrm{y}$ sujeto $14(\mathrm{t}=-2.165 ; p=0.031)$ del grupo experimental. El grupo control presentó modificaciones de los índices $\mathrm{H}$ temporales derechos en tres sujetos (42.9\%) en la segunda medición (uno aumentó y dos disminuyeron) y en el grupo experimental cinco suje- 


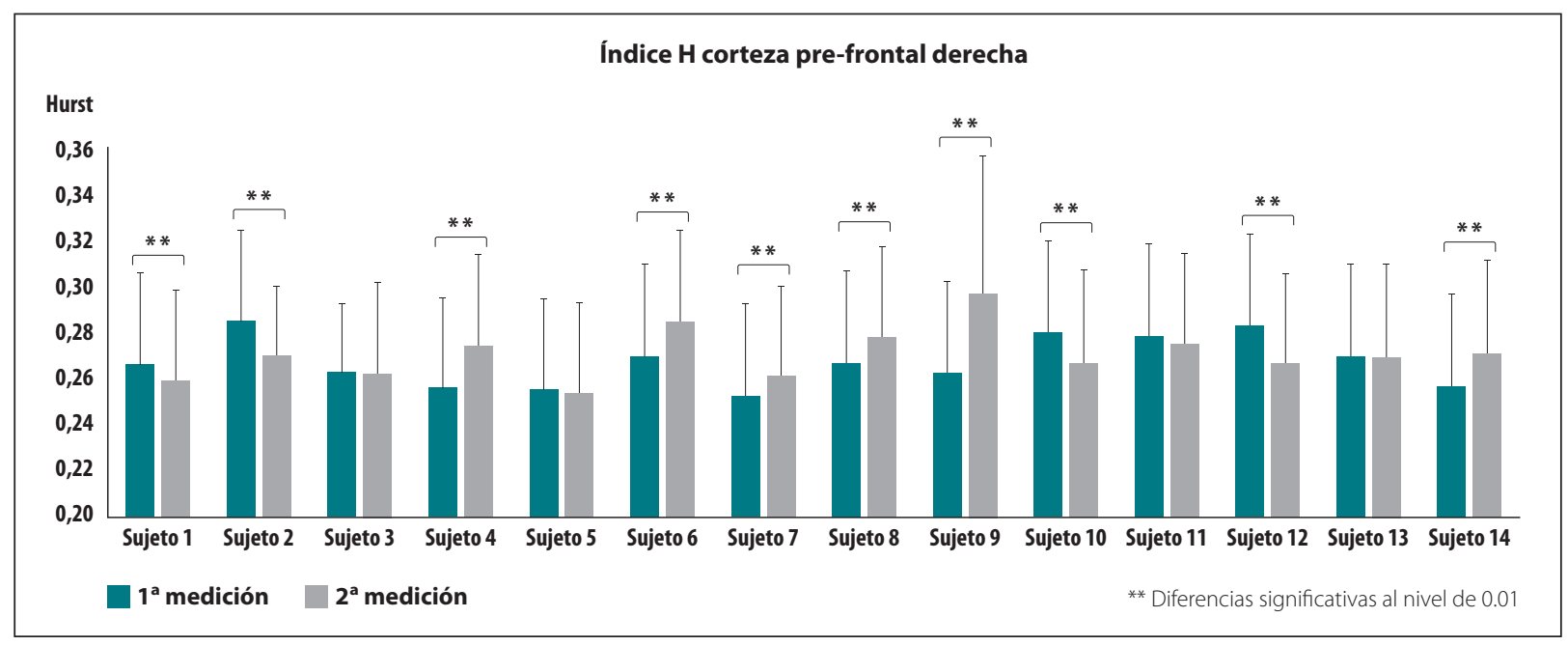

Figura 2. Comparaciones de las medias de los índices de Hurst de la corteza prefrontal izquierda de los sujetos del grupo control (sujeto 1 al 7 ) y del grupo experimental (sujeto 8 al 14) durante los 8 minutos del test de atención en su primera y segunda medición.

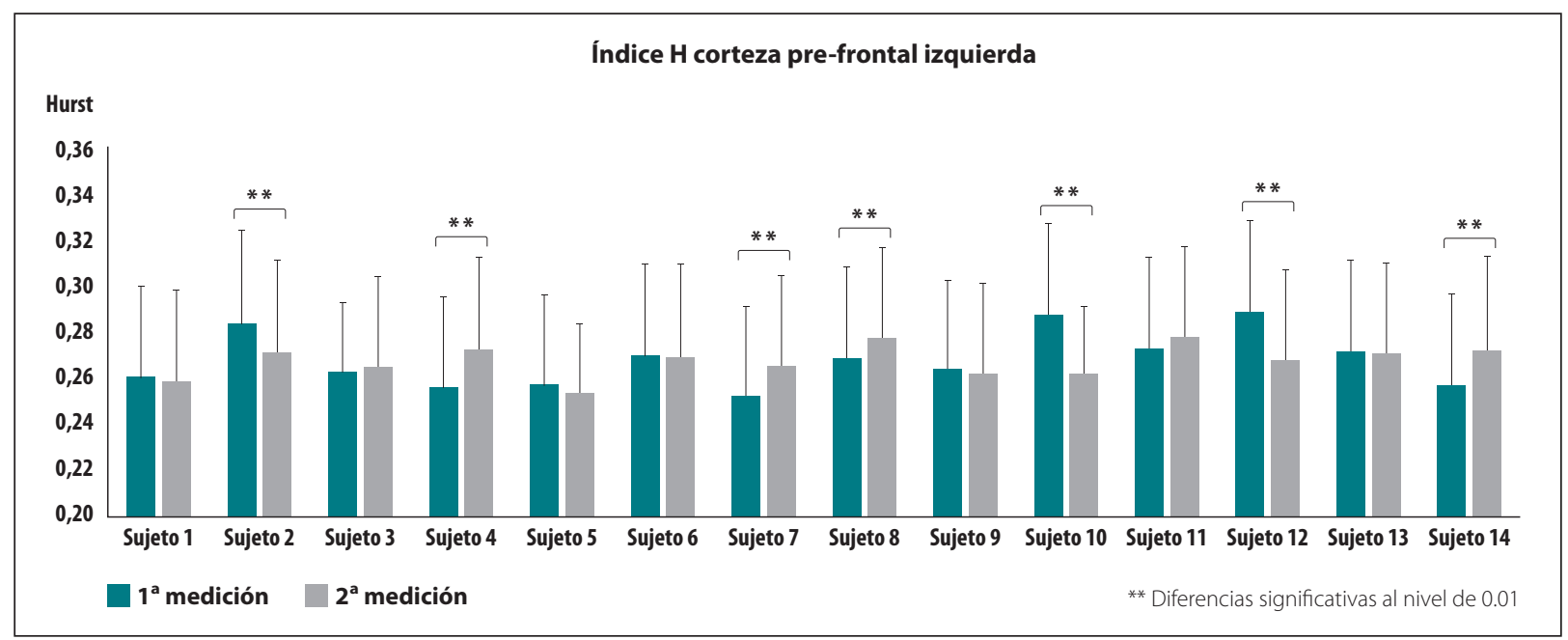

Figura 3. Comparaciones de las medias de los índices de Hurst de la corteza prefrontal derecha de los sujetos del grupo control (sujeto 1 al 7) y del grupo experimental (sujeto 8 al 14) durante los 8 minutos del test de atención en su primera y segunda medición.

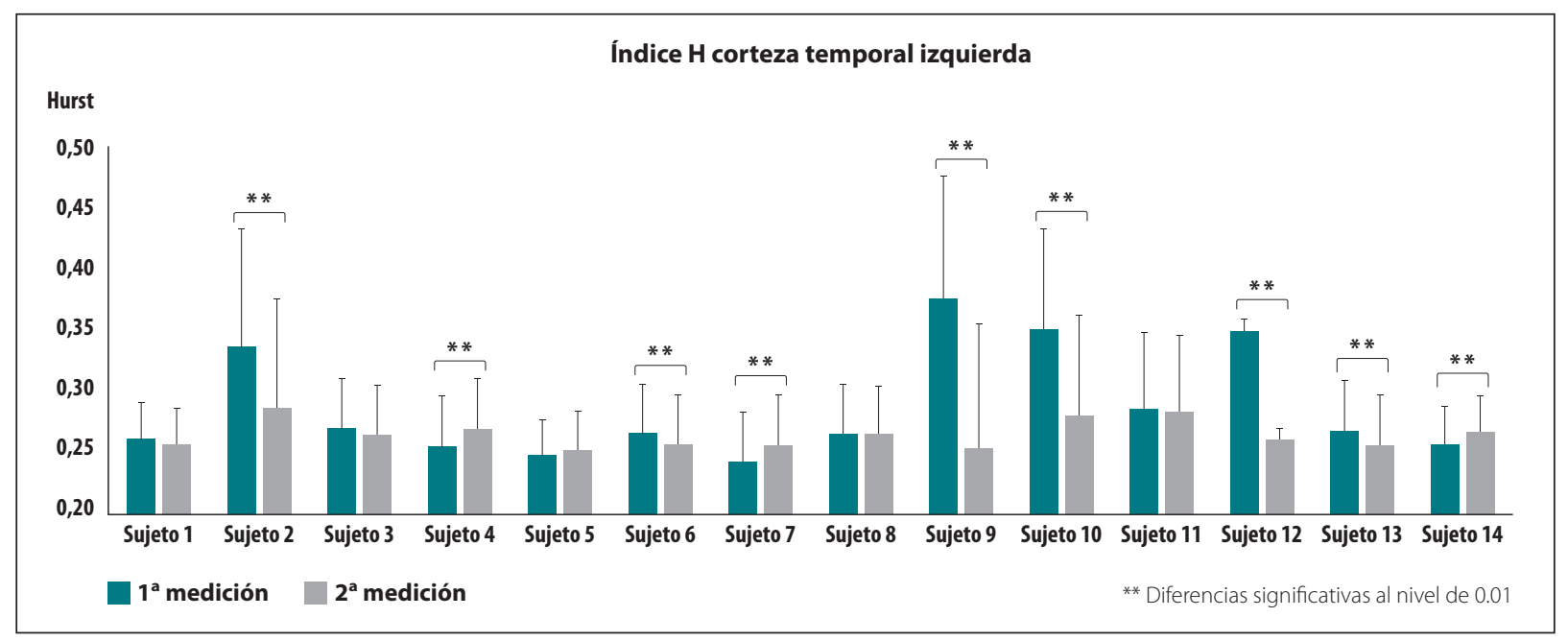

Figura 4. Comparaciones de las medias de los índices de Hurst de la corteza temporal izquierda de los sujetos del grupo control (sujeto 1 al 7 ) y del grupo experimental (sujeto 8 al 14) durante los 8 minutos del test de atención en su primera y segunda medición. 


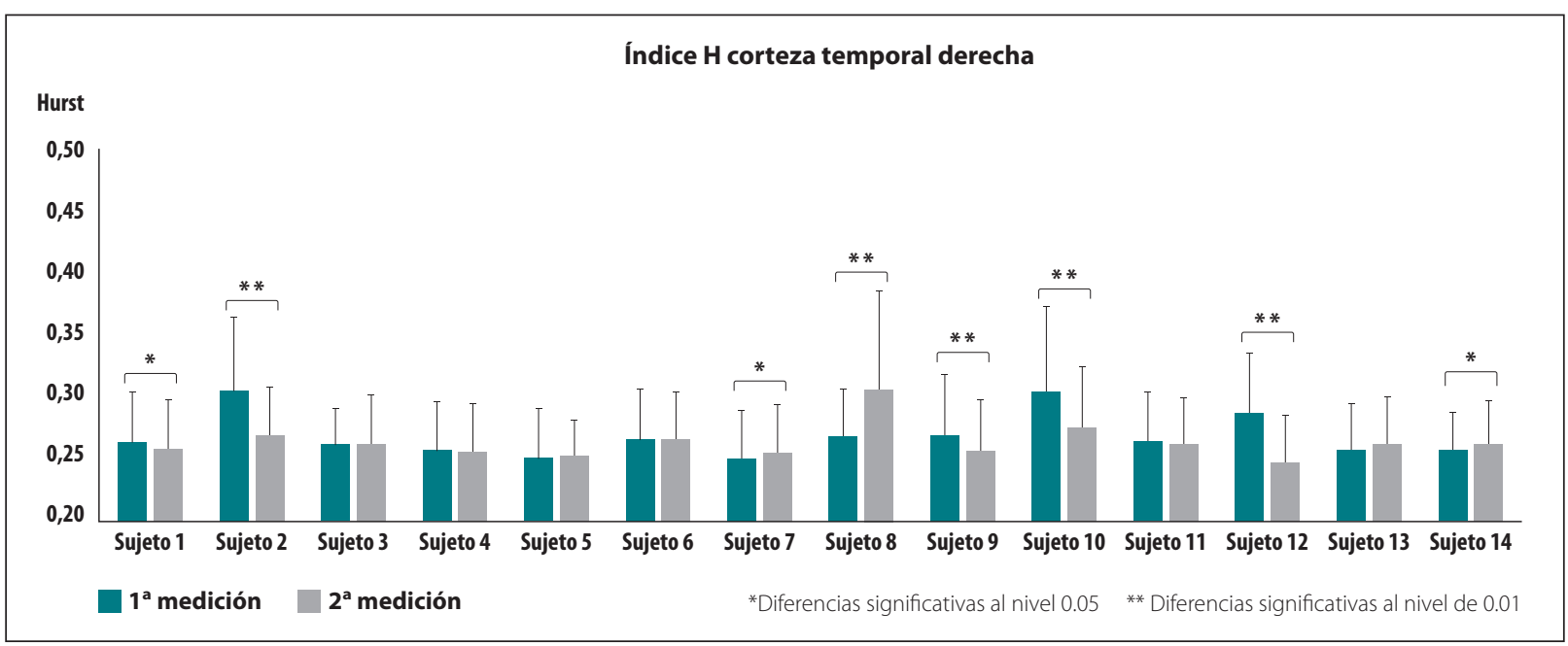

Figura 5. Comparaciones de las medias de los índices de Hurst de la corteza temporal derecha de los sujetos del grupo control (sujeto 1 al 7) y del grupo experimental (sujeto 8 al 14) durante los 8 minutos del test de atención en su primera y segunda medición.

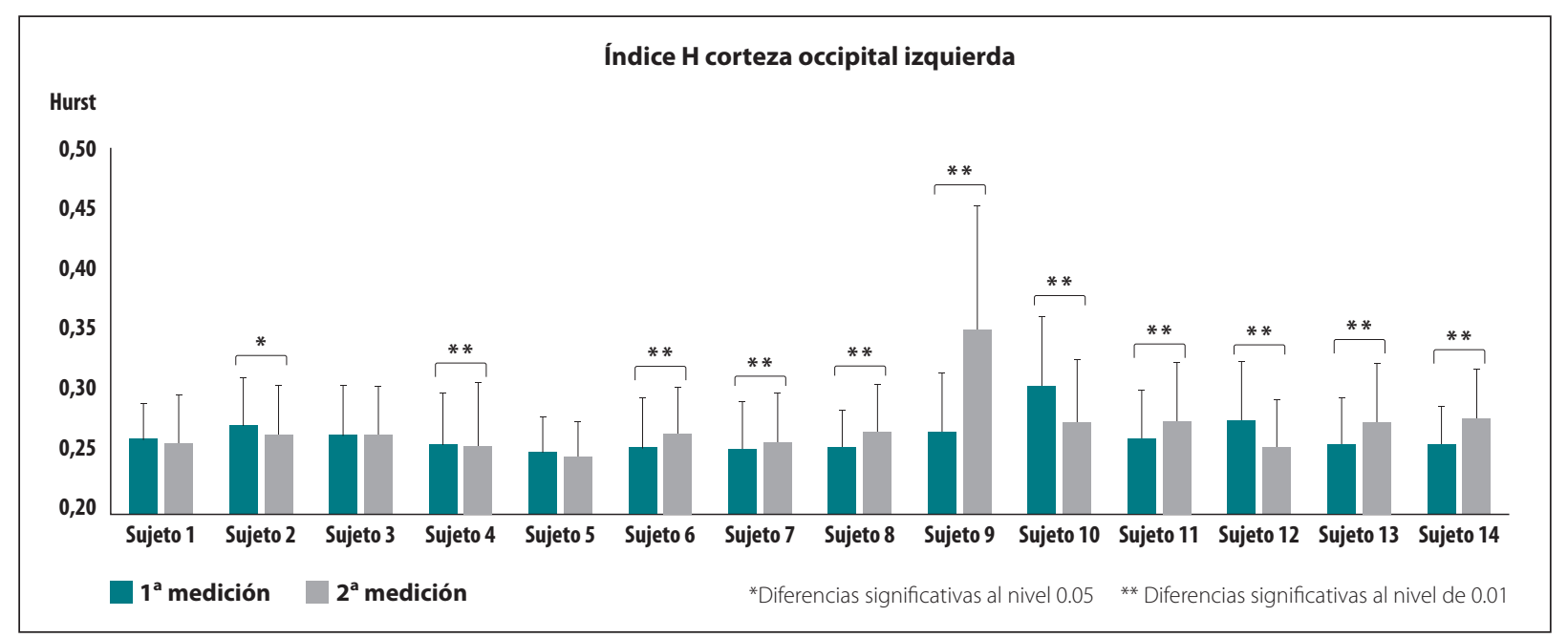

Figura 6. Comparaciones de las medias de los índices de Hurst de la corteza occipital izquierda de los sujetos del grupo control (sujeto 1 al 7 ) y del grupo experimental (sujeto 8 al 14) durante los 8 minutos del test de atención en su primera y segunda medición.

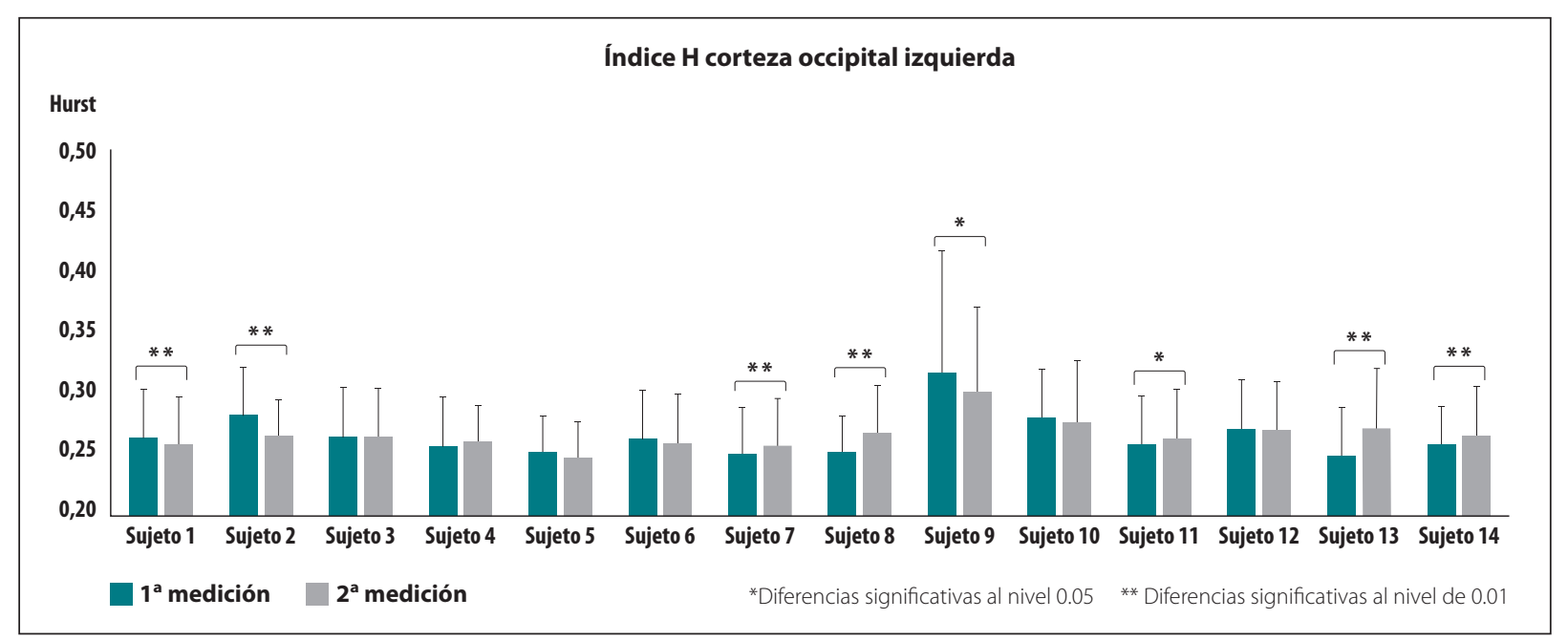

Figura 7. Comparaciones de las medias de los índices de Hurst de la corteza occipital derecha de los sujetos del grupo control (sujeto 1 al 7) y del grupo experimental (sujeto 8 al 14) durante los 8 minutos del test de atención en su primera y segunda medición. 


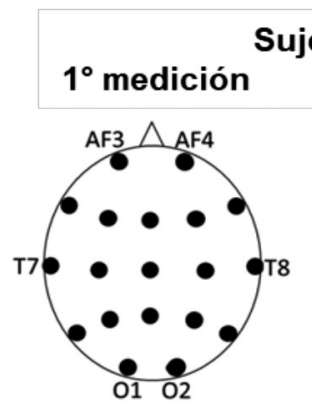

Sujeto 1 $2^{\circ}$ medición

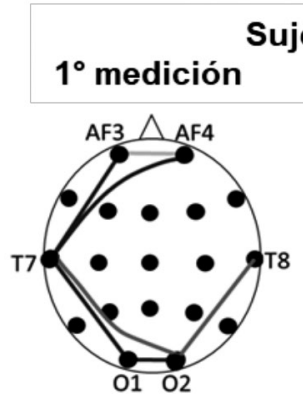

Sujeto 3

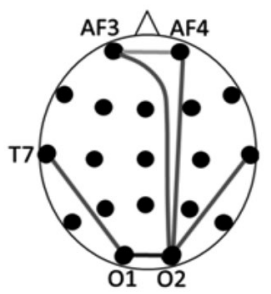

$2^{\circ}$ medición

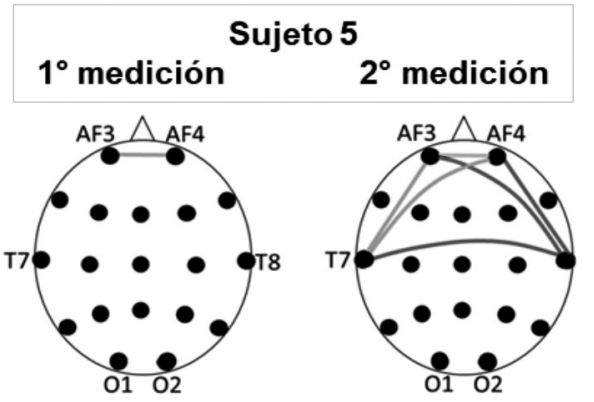

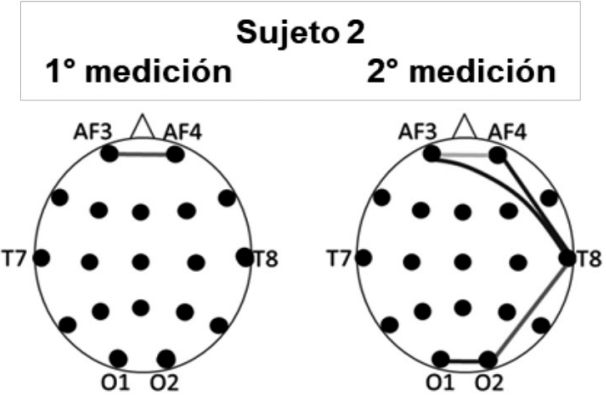

Sujeto 4
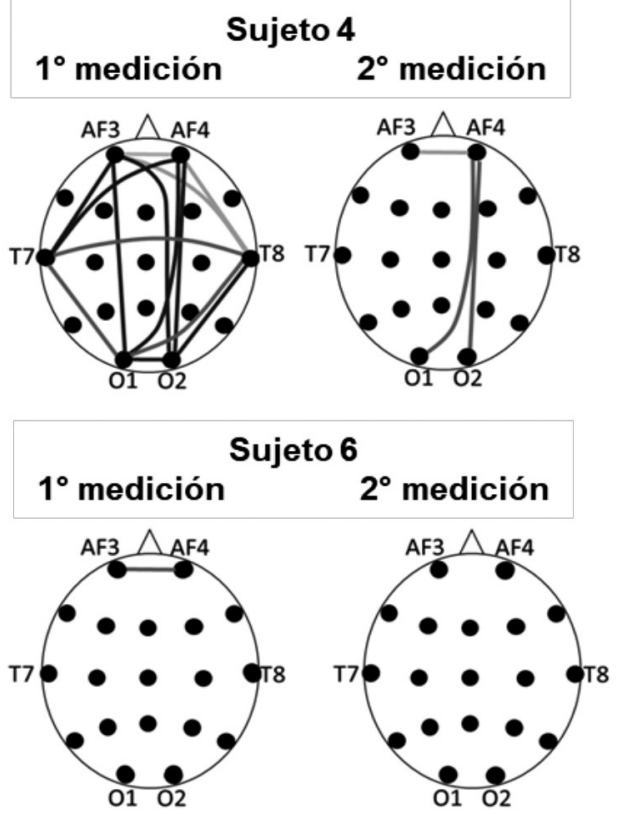

\section{Sujeto 7}

$1^{\circ}$ medición

$2^{\circ}$ medición
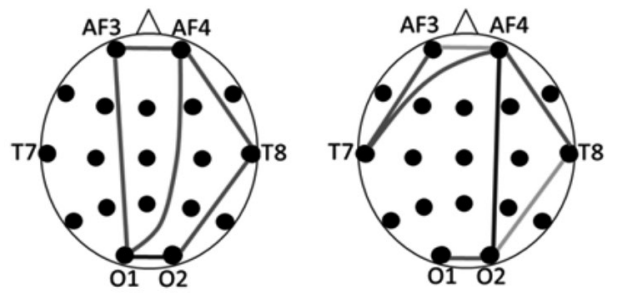

$R=0,900-0,999$

$R=0,800-0,899$

$R=0,700-0,799$

$R=0,600-0,699$

Figura 8. Correlaciones entre las medias de los índices de Hurst de cada corteza cerebral estudiada de los sujetos del grupo control durante los 8 minutos del test de atención en su primera y segunda medición.

tos $(71.4 \%)$ mostraron modificaciones tras el ejercicio físico (uno aumentó y cuatro disminuyeron), sin que existan diferencias significativas entre ambos grupos $(\mathrm{Z}=-1.125)$.

En las figuras 6 y 7 se observan las comparaciones de la actividad eléctrica utilizando el índice de Hurst de la corteza occipital durante las dos mediciones realizadas. Se puede apreciar que existen diferencias significativas en la región occipital izquierda (O1) en el sujeto 2 ( $\mathrm{t}=2.373 ; p=0.018)$, el sujeto $4(\mathrm{t}=-3.906$; $p=0.000)$, el sujeto $6(\mathrm{t}=-3.980 ; p=0.000)$ y el sujeto 7 $(\mathrm{t}=-2.795 ; p=0.005)$ del grupo control; $\mathrm{y}$ en el sujeto $8(\mathrm{t}=-4.681 ; p=0.000)$, sujeto $9(\mathrm{t}=-14.991 ; p=0.000)$, sujeto $10(\mathrm{t}=6.169 ; p=0.000)$, sujeto $11(\mathrm{t}=-4.411$; $p=0.000)$, sujeto $12(\mathrm{t}=6.681 ; p=0.000)$, sujeto $13(\mathrm{t}=-$ $5.751 ; p=0.000)$ y sujeto $14(\mathrm{t}=-8.059 ; p=0.000)$ de 


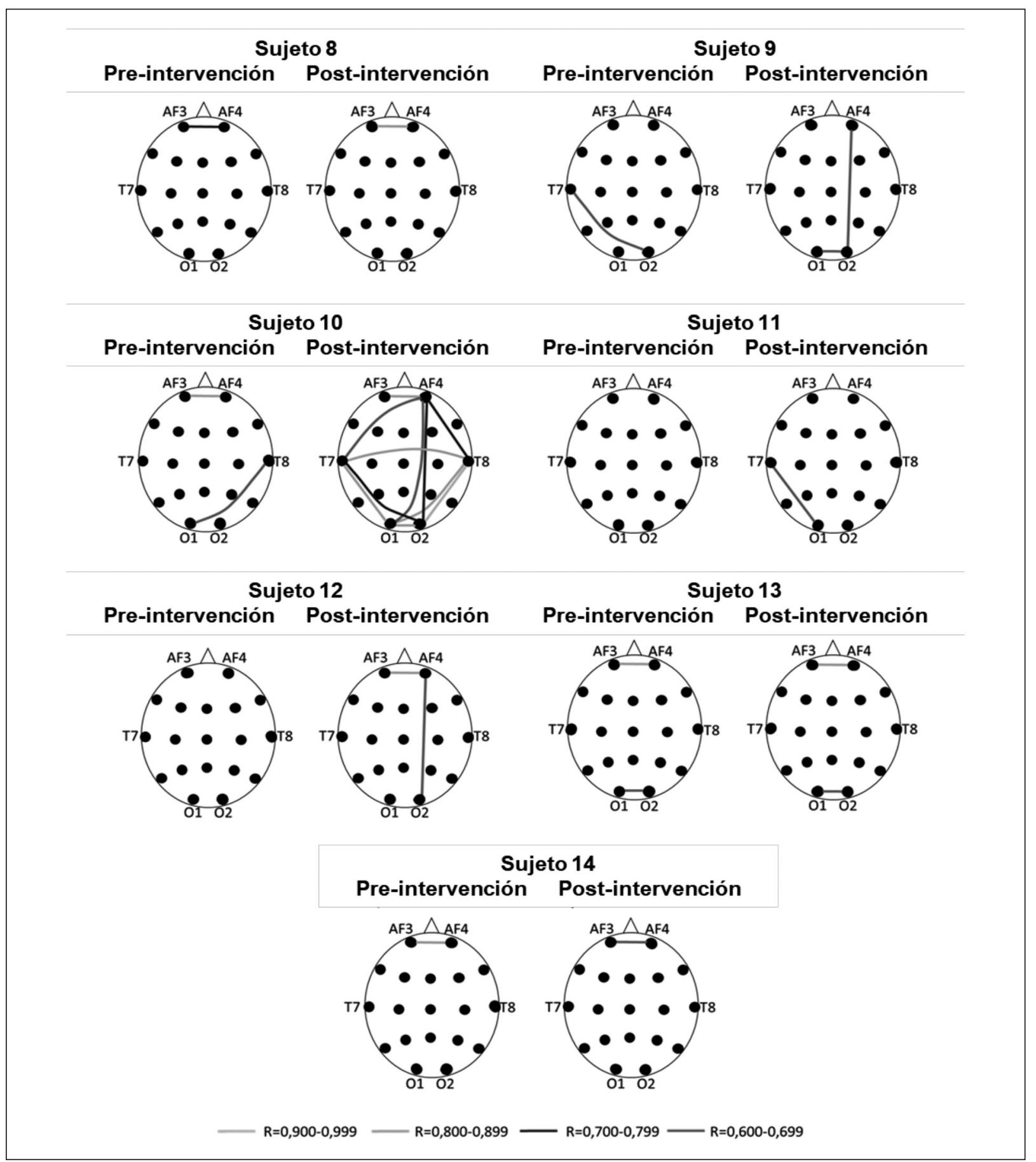

Figura 9. Correlaciones entre las medias de los índices de Hurst de cada corteza cerebral estudiada de los sujetos del grupo experimental durante los 8 minutos del test de atención en su medición pre y post ejercicio físico.

grupo experimental. El grupo control presentó modificaciones de los índices $\mathrm{H}$ occipitales izquierdos en cuatro sujetos $(57.1 \%)$ en la segunda medición (tres aumentaron y uno disminuyó) y en el grupo experimental los siete sujetos (100\%) mostraron modificaciones tras el ejercicio físico (cinco aumentaron y dos disminuyeron), existiendo diferencias significativas entre ambos grupos $(Z=-2.293)$.
En la región occipital derecha (O2) existen diferencias significativas en el sujeto $1(\mathrm{t}=2.870 ; p=0.004)$, sujeto $2(\mathrm{t}=6.366 ; p=0.000)$ y sujeto $7(\mathrm{t}=-2.695$; $p=0.007)$ del grupo control, y el sujeto $8(\mathrm{t}=-6.589$; $p=0.000)$, sujeto $9(\mathrm{t}=2.311 ; p=0.021)$, sujeto $11(\mathrm{t}=-$ $1.998 ; p=0.046)$, sujeto $13(\mathrm{t}=-7.224 ; p=0.000)$ y sujeto $14(\mathrm{t}=-2.924 ; p=0.004)$ del grupo experimental. El grupo control presentó modificaciones de los índices 
H prefrontales derechos en tres sujetos (42.9\%) en la segunda medición (uno aumentó y dos disminuyeron) y en el grupo experimental cinco sujetos (71.4\%) mostraron modificaciones tras el ejercicio físico (cuatro aumentaron y uno disminuyó), sin que existan diferencias significativas entre ambos grupos $(Z=-1.125)$.

En las figuras 5 y 6 se pueden observar las correlaciones de Pearson entre la corteza prefrontal, temporal y occipital para la atención sostenida, de los 14 sujetos pre y post intervención. Se puede apreciar que en el grupo control los sujetos 1, 2, 5 y 6 tienen pocas correlaciones pre-intervención y solo el sujeto 6 no tiene correlaciones entre las cortezas post-intervención. También se puede observar que los sujetos del grupo experimental tienen menos correlaciones respecto al grupo control. Al analizar los resultados post intervención del ejercicio físico podemos notar que existen dos sujetos, el 11 y el 14, que tienen menos sincronización cerebral antes de la intervención, es decir, disminuyeron sus correlaciones o la utilización de varias cortezas para resolver la misma tarea cognitiva.

En el grupo control todos los sujetos (100\%) presentaron variaciones en la cantidad de correlaciones, aumentando el número en la mayoría de los sujetos. Por su parte, el grupo experimental presentó en 4 sujetos (57.2\%) variaciones en la cantidad de correlaciones, aumentando en los cuatro casos. Existen diferencias significativas entre ambos grupos tras la intervención con ejercicio físico, siendo el grupo experimental quién presenta menos modificaciones $(Z=2.289)$.

Finalmente, la cantidad de correlaciones sobre 0.600 y los puntajes finales (IGAP) de la prueba de atención sostenida, tanto en las mediciones pre y post-intervención, no presentan un patrón que permita relacionarlas. Por ejemplo, los sujetos del cuartil más alto de puntajes IGAP obtuvieron en general bajo número de correlaciones, misma situación que ocurre en el cuartil más bajo de puntajes IGAP.

\section{Discusiones}

Los valores de índices de Hurst encontrados en este estudio son menores a 0.5 , lo que muestra una dinámica antipersistente de la actividad neurofisiológica durante la resolución de una prueba de atención sostenida. Esto permite inferir que la actividad eléctrica cerebral frente a factores externos y la propia actividad interna tiende a modificarse a cada segundo, cambiando su comportamiento, a través de un efecto de memoria de corto plazo, durante la resolución de una prueba de atención. Parece ser que los procesos cognitivos aquí estudiados, en una ventana temporal pequeña (un segundo), también operen a una escala de tiempo reducido, lo que haría variar continuamente la relación orden/caos del sistema como una forma de incorporar recursos informáticos que permitan ejecutar la tarea. Con anterioridad se ha observado que los procesos mentales pueden trabajar a diferentes escalas temporales, ejerciendo un control momento a momento (en uno o tres segundos) y un control de largo plazo como resultado del total de la ejecución de la tarea (30 segundos o minutos), por lo tanto, al igual que en la imaginación de una tarea motriz (Díaz, Maureira \& Córdova, 2017) parece ser que durante los procesos atencionales existe, al menos, un proceso antipersistente que permitirá al cerebro controlar la actividad cognitiva momento a momento durante la ejecución de la prueba.

En relación con lo anterior, la aplicación de 30 minutos de ejercicio físico aeróbico parece no afectar estos procesos neurofisiológicos, pese a una mejora en la ejecución de la prueba atencional. Solo se observa una aumento en la variación de los índices $\mathrm{H}$ en la corteza temporal derecha (asociada a la memoria) en el grupo experimental, aunque no es significativa en relación al grupo control, y en la corteza occipital izquierda (asociada a la visión del ojo derecho) del grupo experimental, aumentando el valor $\mathrm{H}$ en cinco de ellos, lo que da cuenta de un aumento en el caos del sistema y de la impredecibilidad del mismo, tal vez porque la atención visual del ojo derecho (toda la muestra es diestra) se asocie con mejores parámetros de búsqueda de las figuras requeridas durante la prueba, lo cual conlleve a mejores puntuaciones del grupo experimental. Esto es similar a lo estudiado por Flores, Maureira, Díaz, Navarro, Gavotto \& Matheu (2019) con la atención selectiva, donde se han encontrado patrones pre y post ejercicio físico e identificado diferencias significativas tras la intervención en la corteza temporal derecha.

El número de correlaciones entre las tres regiones cerebrales estudiadas tiende a ser bajo en la mayoría de los sujetos, lo cual podría explicarse por la asignación de recursos a procesos más específicos, donde cada área se encarga de elementos individualizados que permitan resolver la tarea (Díaz, Maureira, Córdova \& Palominos, 2017). Tras el ejercicio físico el número de correlaciones se mantiene estable en el $50 \%$ de los sujetos, situación que disminuye a cero en el grupo control. Esto resulta interesante, ya que podría ser que después de la actividad física el cerebro continúa con actividad independiente entre diversas regiones, encargándose cada área de la resolución de un problema particular de la prueba neuropsicológica; en cambio los cerebros del grupo control tienden a aumentar su colaboración inter-regiones en la segunda medición, 
tal vez con la finalidad de mejorar su desempeño en la prueba, mismos resultados que obtienen los experimentales (mejor puntaje en la prueba de atención), pero sin la necesidad de aumentar el trabajo sincrónico y, por lo tanto, con un menor consumo energético, dando lugar a un sistema más eficiente. El ejercicio físico podría ayudar, mediante diferentes estrategias cerebrales (aumento o disminución de los índices $\mathrm{H}$ ) y tendencia a mantener el número de correlaciones entre la actividad de diversas regiones de la corteza cerebral, a mejorar la resolución de problemas cognitivos.

Los estudios de la actividad eléctrica cerebral antes y después de intervenciones con ejercicio físico son escasos y, en general, los trabajos se concentran en la amplitud de ondas, intensidades, potenciales evocados, etc. que registra el EEG, pero cuando estas señales son analizadas con matemática no lineal la literatura solo muestra un trabajo al respecto (Flores et al., 2019).

\section{Conclusiones}

En relación con las comparaciones intra-hemisféricas de la corteza prefrontal, temporal y occipital izquierdas y derechas se pudo observar que durante la resolución del test de atención sostenida pre y post intervención del ejercicio físico no existen diferencias significativas en ninguno de los grupos evaluados. Al realizar las correlaciones de Pearson para los sujetos del grupo control y experimental pre y post intervención se pudo observar que cada sujeto tuvo variaciones en cada medición, es decir, resolvieron el test de manera diferente cada vez, existiendo diferentes números de correlaciones. Al realizar la prueba de proporciones para comparar las variaciones (aumento o disminución de correlaciones) los valores $\mathrm{Z}$ mostraron diferencias significativas, donde el grupo experimental presentó menos modificaciones en la cantidad de correlaciones postejercicio físico.

Si bien es una limitación importante es la muestra, no se puede olvidar que la actividad cerebral durante la percepción y la resolución de problemas cognitivos es única, es decir, cada cerebro y, por ende, cada persona, tiene su propia estrategia para enfrentar un problema. Esta situación complica mucho la extrapolación de resultados a una población, teniendo que analizarse cada caso en forma independiente.

Son necesarias más investigaciones profundizando la relación orden/caos de la actividad neurofisiológica durante la resolución de pruebas cognitivas. En cuanto la atención, sería interesante estudiar otras ventanas temporales de índices de Hurst para corroborar la utilización de diversos procesamientos en paralelo durante los procesos mentales que llevan a la resolución de problemas cognitivos. También es necesario estudiar otros grupos etarios, test cognitivos, tipos de ejercicios o entrenamientos e incluir estudios en damas para diversificar los resultados aquí encontrados. 


\section{BIBLIOGRAFÍA}

Altenburg, T., Chinapaw, M. \& Singh, A. (2016). Effects of one versus two bouts of moderate intensity physical activity on selective attention during a school morning in Dutch primary school-children: A randomized controlled trial. Journal of Science and Medicine in Sport, 19(10), 820-824. doi:10.1016/j.jsams.2015.12.003

Alves, C., Tessaro, V., Teixeira, L., Murakava, K., Roschel, H., Gualano, B., et al. (2014). Influence of acute high-intensity aerobic interval exercise bout on selective attention and short-term memory tasks. Perceptual and Motor Skills, 118(1), 63-72. doi:10.2466/22.06. PMS.118k10w4

Babiloni, C., Infarinato, F., Marzano, N., Lacobani, M., Dassú, F., Soricelli, A., et al. (2011). Intra-hemispheric functional coupling of alpha rhythms is related to golfer's performance: A coherence EEG study. International Journal of Psychopjysiology, 82(3), 260-268. doi:10.1016/j. ijpsycho.2011.09.008

Bullock, T. \& Giesbrecht, B. (2014). Acute exercise and aerobic fittness influence selective attention during visual search. Frontiers in Psychology, 5, 1290. doi:10.3389/fpsyg.2014.01290

Buzsaki, G. (2006). Rhythms of the brain. London: Oxford University Press.

Cotman, C. \& Berchtold, N. (2002). Exercise: a behavioral intervention to enhance brain health and plasticity. Trends Neuroscience, 25, 295301. doi:10.1016/S0166-2236(02)02143-4

Daronch, C., De Souza, R., Spindolab, M., Vaza, M., De Oliveira, L. \& Geremiad, J. (2016). Bailarinas x voleibolistas: efeito de diferentes treinamentos motores sobre o sinal eletroencefalográfico. Rev. Bras. Ciênc. Esporte, 38(4), 384-391. doi:10.1016/j.rbce.2016.02.007

Díaz, H., Maureira, F. \& Córdova, F. (2017). Temporal scaling and interindividual hemispheric asymmetry of chaos estimation from EEG time series. Procedia Computer Science, 122, 339-345. doi:10.1016/j. procs.2017.11.378

Díaz, H., Maureira, F. \& Córdova, F. (2018). Time series of closed and open eyes EEG conditions reveal differential characteristics in the temporality of linear and non-linear analysis domain. Procedia Computer Science, 139, 570-577. doi:10.1016/j.procs.2018.10.208

Díaz, H., Maureira, F., Córdova, F. \& Palominos, F. (2017). Long-range linear correlation and nonlinear chaos estimation differentially characterizes functional conectivity and organization of the brain EEG. Procedia Computer Science, 122, 857-864. doi:10.1016/j. procs.2017.11.447

Estévez, A., García, C. \& Junque, C. (1997). La atención: una compleja función cerebral. Revista de Neurología, 25, 1989-1997.

Fan J, McCandliss B, Fossella J, Flombaum J. \& Posner M. (2005). The activation of attentional networks. Neuroimage 2005, 26: 471-479. doi:10.1016/j.neuroimage.2005.02.004

Flores, F., Maureira, F., Díaz, H., Navarro, B., Gavotto, O. y Matheu, A. (2019). Efectos de una sesión de ejercicio físico sobre la actividad neurofisiológica durante la resolución de una prueba de atención selectiva. Retos, 36, 390-396.
Fuenmayor, G. \& Villasmil, Y. (2008). La percepción, la atención y la memoria como procesos cognitivos utilizados para la comprensión textual. Revista de Artes y Humanidades UNICA, 9(22), 187-202.

García, J. (1997). Psicología de la atención. Madrid: Síntesis Psicología.

Hirata A. \& Castro-Alamancos M. (2010). Neocortex network activation and deactivation states controlled by the thalamus. J Neurophysiol, 103, 1147-1157. doi:10.1152/jn.00955.2009

Hsieh, S., Chang, Y., Fang, C. \& Hung, T. (2016). Acute resistance exercise facilitates attention control in adult males without an agemoderating effect. Journal Sport Exercise Psychology, 38(3), 247-254. doi:10.1123/jsep.2015-0282

Iuliano, E., di Cagno, A., Aquino, G., Fiorilli, G., Mignogna, P., Calcagno, G., et al. (2015). Effects of different types of physical activity on the cognitive functions and attention in older people: A randomized controlled study. Experimental Gerontology, 70, 105-110. doi:10.1016/j. exger.2015.07.008

León-Carrión, J. (1995). Manual de neuropsicología humana. Madrid: siglo XXI

Ludyga, S., Gronwald, T. \& Hottenrott, K. (2016). Effects of high vs low cadence training on cyclists' brain cortical activity during exercise. Journal of Science and Medicine in Sport, 19(4), 342-347. doi:10.1016/j. jsams.2015.04.003

Maureira, F. (2017). ¿Qué es la intligencia? Madrid: Bubok Publishing.

Maureira, F. (2018). Principios de Neuroeducación Física. Madrid: Bubok Publishing.

Maureira, F. \& Flores, E. (2018). Electroencefalografía (EEG) y diversas manifestaciones del movimiento: una revisión del 2000 al 2017. EmásF, Revista digital de Educación Física, 9(51), 48-63.

Maureira, F. \& Flores, E. (2016). Neuropsicobiología para estudiantes de educación. Valencia: Obrapropia.

Pikovsky, A., Rosenblum, M. \& Kurths, J. (2001). Synchronization: a universal concept in nonlinear sciences. Cambridge: Cambridge University Press.

Rogerson, M. \& Barton, J. (2015). Effects of the visual exercise environments on cognitive directed attention, energy expenditure and perceived exertion. International Journal Environ Research Public Health, 12(7), 7321-7336. doi:10.3390/ijerph120707321

Vidoni, E., Johnson, D., Morris, J., Van Sciver, A., Greer, C., Billinger, S., et al. (2015). Dose-response of aerobic exercise on cognition: a community-based, pilot randomized controlled trial. PLoS One, 10(7): e0131647. doi:10.1371/journal.pone.0131647

Wilmore, J. \& Costill, D. (2004). Fisiología del esfuerzo y del deporte. Barcelona: Paidotribo.

Voss, M., Prakash, R., Erickson, K., Basak, C., Chaddock, L., Kim, J., et al. (2010). Plasticity of brain networks in a randomized intervention trial of exercise training in older adults. Frontiers in Aging Neuroscience, 2, 1-17. doi:10.3389/fnagi.2010.00032 